\title{
ANALYTICAL APPROXIMATION TO THE TURN-TO-TURN QUENCH PROPAGATION
}

\author{
G. LÓPEZ \\ Superconducting Super Collider Laboratory * \\ 2550 Beckleymeade Avenue \\ Dallas, Texas 75237
}

September 1990

\section{DISCLAIMER}

\begin{abstract}
This report was prepared as an account of work sponsored by an agency of the United States Government. Neither the United States Government nor any agency thereuf, nor any of their employees, makes any warranty, express or implied, or assumes any legal liability or responsi. bility for the accuracy, completeness, or usefulness of any information, apparatus, product, or process disclosed, or represents that its use would not infringe privately owned rights. Reference herein to any specific commercial product, process, or service by trade name, trademark, manufacturer, or otherwise does not necessarily constitute or imply its endorsement, recommendation, or favoring by the United States Government or any agency thereof. The views and opinions of authors expressed herein do not necessarily state or reflect those of the United States Government or any agency thereof.
\end{abstract}

\footnotetext{
* Operated by the Universities Research Association, for the U. S. Department of Energy under Contract No. DE-AC02-89ER40486.
} 
SSCL-309

\title{
ANALYTICAL APPROXIMATION TO THE TURN-TO-TURN QUENCH PROPAGATION
}

\author{
G. LÓPEZ \\ Superconducting Super Collider Laboratory * \\ 2550 Beckleymeade Avenue \\ Dallas, Texas 75237
}

September 1990

\begin{abstract}
Using a gross approximation for the flow of heat in a one-dimensional model. an analytical expresion is obtained for the turn-to-turn time delay to quench other nearby conductors. This expression agrees qualitatively with the experimental data of the SSC $R \& D$ dipole magnets.
\end{abstract}

* Operated by the Universities Research Association, for the U. S. Department of Energy under Contract No. DE-AC02-89ER40486. 


\section{INTRODUCTION}

The turn-to-turn time delay, for quenching other conductors of the coil in a magnet, is an important parameter which measures the transversal quench propagation; consequently, it measures how fast the stored energy of the magnet is spread in the coil. This is very closely related to the hot-spot temperature (where a quench first appears) in the coil, establishing the fact that for faster energy spread, there is a lower hot-spot temperature and vice versa.

Quench simulation programs use the turn-to-turn time delay ${ }^{(1,2)}$ or the transversal quench velocity $^{(2)}$ to take account of the spread of the energy in the coil; the former is taken from experimental data and the last one from theoretical assumptions. The common transversal quench propagation used is given by

$$
v_{\perp}=\sqrt{\frac{k_{i}}{k_{c}}} v_{\|}
$$

where $k_{i}$ is the thermal conductivity of the insulation which covers the conductor, $k_{c}$ is the thermal conductivity of the conductor, $v_{\|}$is the axial quench velocity. This expression brings about the following turn-to-turn time delay for quenching

$$
\tau_{d}=\sqrt{\frac{k_{c}}{k_{i}}} \frac{L}{v_{\|}}
$$

where $L$ is the insulation thickness between conductors. It has been established that the above relations are not satisfied for the $40 \mathrm{~mm}$ SSC R\&D Dipole Magnets, and there is a general opinion that there rnust be other mechanism involved ${ }^{(3)}$ in this phenomenon. An explanation of this time delay, or at least an approximation of it, would be useful for quench simulations, since a confident result cculd be obtained to predict the quench behavior of the magnet before making a full test experiment. In this report, an analytical approximation is used in a one dimensional model for the heat propagation, obtaining an analytical expression for this time delay. The values obtained with this expression are compared with the experimental ones ${ }^{(4)}$.

\section{TURN-TO-TURN TIME DELAY}

Consider two superconducting cables separated by insulation, as shown in the Figure 1. When the first conductor (I) quenches at the time $\tau_{1}$, heat is generated which propagates through the insulation to conductor (II), at that time, there would be other quenches. The problem to address in this report is the time it takes the conductor II to quench. 


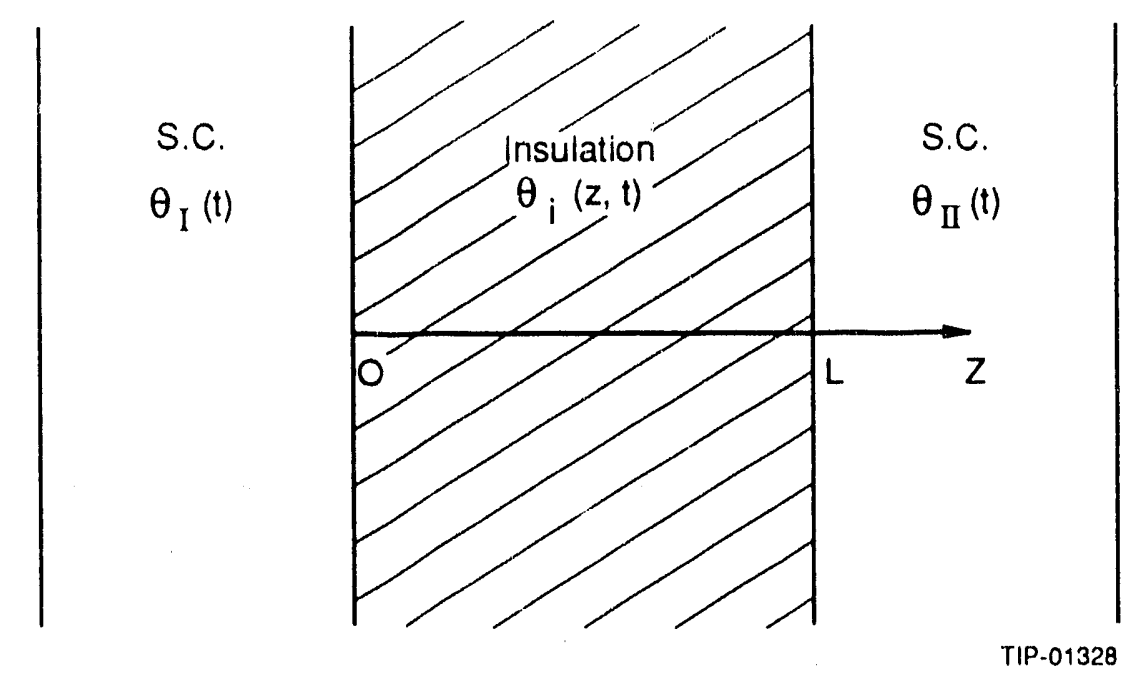

Figure 1. One Dimensional Model. Quench starts in the first conductor and the heat propagates to the second one through the gap of insulation. The temperature at the time $t \leq 0$ is the batch temperature, $\theta_{o}\left(\theta_{I}(\leq 0)=\theta_{i}(z, \leq 0)=\theta_{I I}(\leq 0)=\theta_{o}\right)$.

The temperature in the insulation at any time, is given by solving the following equation

$$
(\delta c)_{i} \frac{\partial \theta_{i}}{\partial t}=-\frac{\partial S}{\partial z}
$$

where $S$ is the flow of heat going into the insulation; $(\delta c)_{i}=(\delta c)\left(\theta_{i}\right)$ is the density multiplied by the specific heat average over the components of the insulation materials; $k_{i}=k\left(\theta_{i}\right)$ is the total thermal conductivity of the insulation. Assuming that the change in the heat flowing along $z$ is proportional to the flow of heat and inverslly proportional to the heat source separation, $z$, the following relation is satisfied

$$
\frac{\partial S}{\partial z}=\frac{S}{z},
$$

where the selected sign in accordance with the reference system chosen. Using (4) in (3), the following nonlinear partial differential equation arises

$$
(\delta c)_{i} \frac{\partial \theta_{i}}{\partial t}+\frac{k_{i}}{z} \frac{\partial \theta_{i}}{\partial z}=0
$$

which can be solved by the Characteristics Method and brings about the solution

$$
C_{2}\left(\theta_{i}\right)-t+\frac{(\delta c)\left(\theta_{i}\right)}{k\left(\theta_{i}\right)} z^{2}=0,
$$


where $C_{2}$ is an arbitrary function of the temperature $\theta_{i}$. Consider that $\theta_{I}(t)$ is the temperature of the first quenched conductor at the time $t$, and that the boundary condition,

$$
\theta_{i}(0, t)=\theta_{l}(t),
$$

is included in the expression (6), then it follows the next solution

$$
C_{2}\left(\theta_{i}(t)\right)=t
$$

Thus, due $\theta_{i}(t)$ is an increasing function with time; $C_{2}$ must be the inverse function of $\theta_{I}$, that is

$$
C_{2}(s)=\theta_{I}^{-1}(s)
$$

for any parameter $s$; in this way, the solution (6) is written in the following implicit form

$$
\theta_{I}^{-1}\left(\theta_{i}\right)-t+\frac{(\delta c)\left(\theta_{i}\right)}{k\left(\theta_{i}\right)} z^{2}=0,
$$

or it can be written as the implicit relation

$$
\theta_{i}=\theta_{I}\left(t-\frac{(\delta c)\left(\theta_{i}\right)}{k\left(\theta_{i}\right)} z^{2}\right)
$$

Assume now, that at the end of the insulation, $z=L$, and at the time $t=\tau_{2}$, the temperature (11) equals the heat generation temperature started in the first conductor, $\theta_{g}$, then the relation (11) is expressed as

$$
\theta_{g}=\theta_{I}\left(\tau_{2}-\frac{(\delta c)\left(\theta_{g}\right)}{k\left(\theta_{g}\right)} L^{2}\right),
$$

but if $\tau_{1}$ is the time at which the heat generation starts in the first conductor, from the relation (12), it would follow the expression

$$
\tau_{1}=\tau_{2}-\frac{(\delta c)\left(\theta_{g}\right)}{k\left(\theta_{g}\right)} L^{2}
$$

Thus, the border of the insulation, $z=L$, will have the temperature $\theta_{g}$ at the time

$$
\tau_{2}=\tau_{1}+\frac{(\delta c)\left(\theta_{g}\right)}{k\left(\theta_{g}\right)} L^{2}
$$

Time, $\tau_{1}$, can then be calculated from

$$
(\delta c)_{c} \frac{d \theta_{I}}{d t}=\rho J^{2}
$$

where $\rho$ is the total resistivity of the conductor materials and $J$ is the current density; $(\delta c)_{\text {. }}$ is the density multiplied by the specific heat averaged over the conductor 
materials. The current and the magnetic field are taken as constants during the process. Integrating (15) with the initial condition $\theta_{I}(0)=\theta_{i}(z, 0)=\theta_{0}$, the next relation follows

$$
\tau_{1}=\frac{1}{J^{2}} \int_{\theta_{0}}^{\theta_{0}} \frac{(\delta c)_{c}}{\rho} d \theta_{l}
$$

Assuming that the characteristics of both superconducting cables are the same, the turnto-tuin time delay is given by

$$
\tau_{d}=\frac{2}{J^{2}} \int_{\theta_{o}}^{\theta_{3}} \frac{(\delta c)_{c}}{\rho} d \theta+\frac{(\delta c)\left(\theta_{g}\right)}{k\left(\theta_{g}\right)} L^{2}
$$

\section{RESULTS AND CONCLUSIONS}

Figure 2 shows the vilues obtained using (17) in comparison with some experimental results ${ }^{(4)}$. For the calcula:ion of $(17)$, the following parameters were assumed

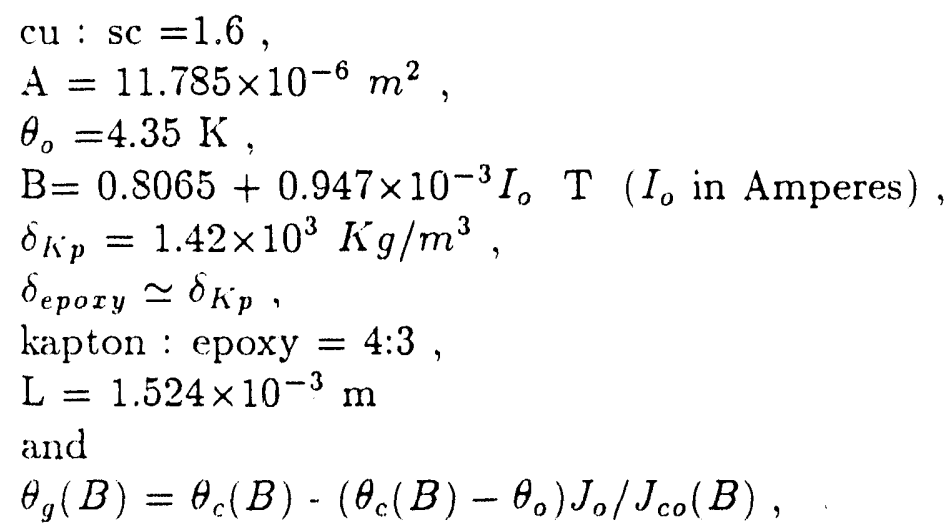

where the critical current temperature, $\theta_{c}$, and the critical current density, $J_{c o}$, are calculated from known expressions ${ }^{(5)}$. The expression (17) gives a gross approximation to the experimental turn-to-turn time delay values for transversal quench propagation and could be used cautiously in quench computer simulations. There is a qualitative difference between expression (17) and (2) related to the separation of the conductors and the thermal conductivity. Although expression (13) is consistent with normal diffusion time between two materials ${ }^{(6)}$, caution is advised, because of the possible inconsistency in this gross approach.

Applying the same expression to the turn-to-turn time delay between wedges provides a time delay much smaller than between the insulation. In this case, the one dimensional model is not valid any more. Due to the high thermal conductivity of copper, heat is spread with the same speed in both directions drastically reducing the time delay for quenching the other conductor. However, other sets of experiments carried out in a $4 \mathrm{~cm}$ SSC R\&D dipole magnet, see refence [7], show that a pure Fourier conduction mechanism (expressions (1) or (17)) for the transversal quench propagation is not enough to understand the transversal quench behavior of these magnets (the turn-to-turn heat. prop). agation is not sequential). 


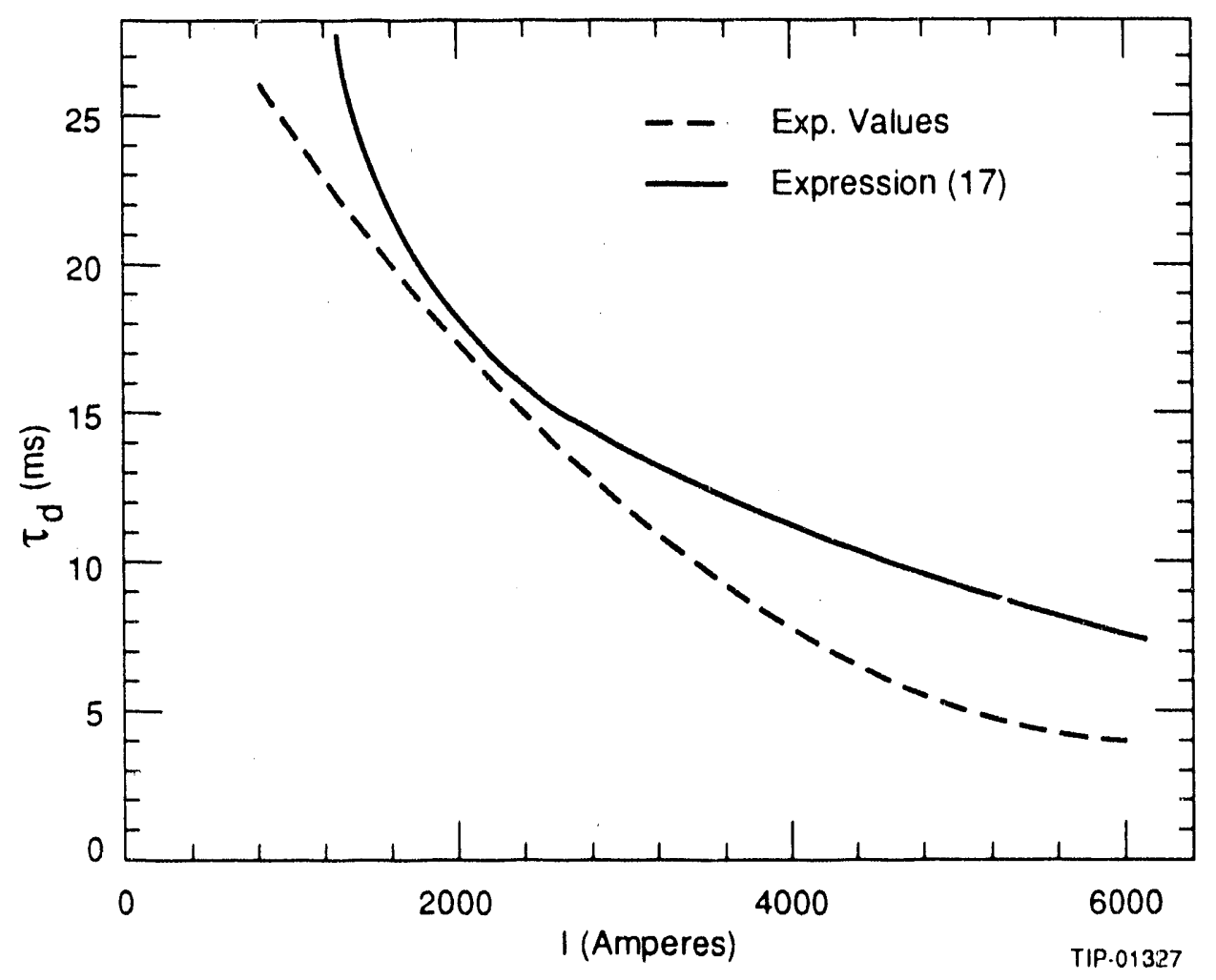

Figure 2. Experimental values for the turn-to-turn time delay in comparison with the expression (17) (contimuous line). 


\section{Acknowledgement}

I wish to thank. Dr. G. Schnitler for useful discutions, Dr. R. Switter and Dr. D. Edwards for their support at the SSC Laboratory.

\section{REFERENCES}

1. G. López and G. Schnitler, SSCL-283, 1990.

2. M. N. Wilson," Superconducting Magnets," Oxford Science Publications, chapter 9, 1983.

3. A. Devred,"Quench Characteristics of Full-Length SSC R\&D Dipole Magnets," SSC-234, 1989.

4. W. B. Sampson, Personal communication 1986.

5. G. Morgan and W. B. Sampson,"New Coefficients for a $J_{c o}(B)$ analytic form," SSC-N-519, 1986.

6. R. P. Shutt,"Estimates of Pressures and Temperatures Generated During Quenches of SSC Magnets," SSC-N-265, 1986.

7. J. Cortella,"Quench Data Analysis of Magnet DD0012 (Parts I,1I and III)," MD-TA-90,91 and 92, 1988. 

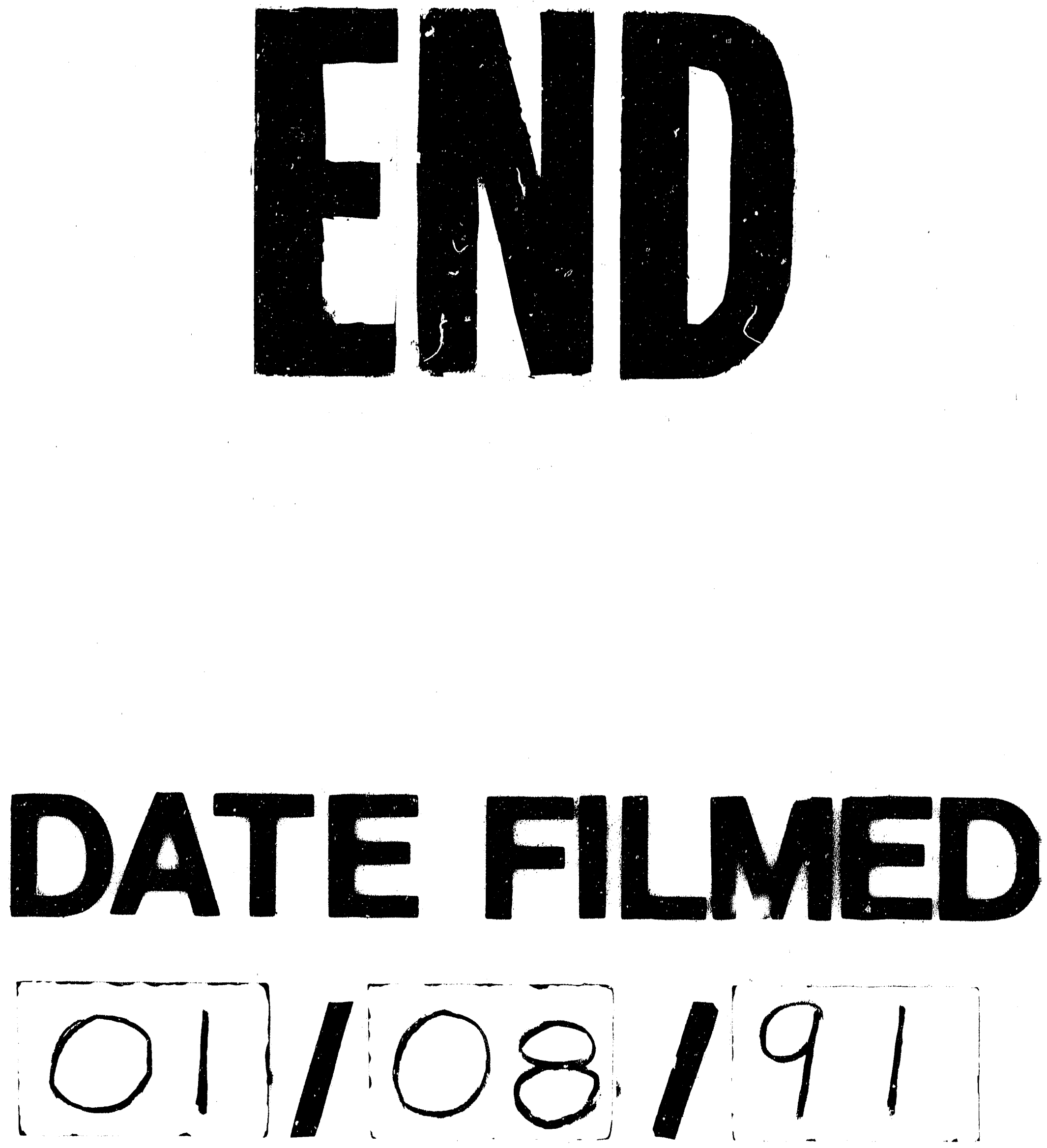


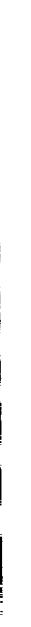

1 